\title{
Indispensability Arguments in favour of Reductive Explanations.
}

\author{
Jeroen Van Bouwel, Erik Weber \& Leen De Vreese ${ }^{1}$ \\ Centre for Logic and Philosophy of Science \\ Ghent University (Belgium)
}

Published in: Journal for General Philosophy of Science 42(1): 33-46.

\section{Contact Address:}

Jeroen Van Bouwel

Centre for Logic and Philosophy of Science

Blandijnberg 2

9000 Gent

Belgium

Email: Jeroen.VanBouwel@UGent.be

\begin{abstract}
:
Instances of explanatory reduction are often advocated on metaphysical grounds; given that the only real things in the world are subatomic particles and their interaction, we have to try to explain everything in terms of the laws of physics. In this paper, we show that explanatory reduction cannot be defended on metaphysical grounds. Nevertheless, indispensability arguments for reductive explanations can be developed, taking into account actual scientific practice and the role of epistemic interests. Reductive explanations might be indispensable to address some epistemic interest answering a specific explanation-seeking question in the most accurate, adequate and efficient way. Just like explanatory pluralists often advocate the indispensability of higher levels of explanation pointing at the pragmatic value of the explanatory information obtained on these higher levels, we argue that explanatory reduction traditionally understood as the contender of pluralism - can be defended in a similar way. The pragmatic value reductionist, lower level explanations might have in the biomedical sciences and the social sciences is illustrated by some case studies.
\end{abstract}

\footnotetext{
${ }^{1}$ The authors would like to thank Jan De Winter, Merel Lefevere and two anonymous referees for their comments on earlier versions of this paper. The research for this paper was supported by the Research Foundation Flanders (FWO) through research projects G.0651.07 and G.0031.09.
} 
Running Head: Indispensability Arguments and Reduction

\section{Indispensability Arguments in favour of Reductive Explanations.}

\section{Abstract:}

Instances of explanatory reduction are often advocated on metaphysical grounds; given that the only real things in the world are subatomic particles and their interaction, we have to try to explain everything in terms of the laws of physics. In this paper, we show that explanatory reduction cannot be defended on metaphysical grounds. Nevertheless, indispensability arguments for reductive explanations can be developed, taking into account actual scientific practice and the role of epistemic interests. Reductive explanations might be indispensable to address some epistemic interest answering a specific explanation-seeking question in the most accurate, adequate and efficient way. Just like explanatory pluralists often advocate the indispensability of higher levels of explanation pointing at the pragmatic value of the explanatory information obtained on these higher levels, we argue that explanatory reductiontraditionally understood as the contender of pluralism - can be defended in a similar way. The pragmatic value reductionist, lower level explanations might have in the biomedical sciences and the social sciences is illustrated by some case studies.

Keywords: biomedical sciences, epistemic interests, explanatory pluralism, reductive explanations, social sciences. 


\section{Introduction.}

In the chapter on reductionism of Thinking about Biology, Stephen Webster defines reductionism as follows:

Let us label this philosophy straight away: it is called reductionism, and can be defined as the belief that the best and most reliable explanations in science depend on a knowledge of components, and are expressed in terms of those components. (2003: 39)

We call reductionism as defined by Webster explanatory reductionism, because it relates to how scientist should behave (what kind of explanations they have to give). In principle, there are two ways to justify explanatory reductionism. The first is to adopt metaphysical reductionism and argue that explanatory reductionism follows from it. The second is to develop indispensability arguments in favour of it. In this paper we argue that the first strategy fails, elaborate the second and show that it does work.

Metaphysical reductionism comes in two kinds: a strong and a weak one. The strong one consists in the view that the only real things in the world are subatomic particles and their interactions. Weak metaphysical reductionism consists in the view that higher level phenomena supervene on subatomic particles and their interactions. In Section 2, we show that neither the strong nor the weak variant of metaphysical reductionism provides an argument for explanatory reductionism. This prepares the ground for the following sections.

In Section 3, we clarify how we try to build indispensability arguments for reductionism, articulating some important characteristics of scientific explanations and introducing the idea of explanatory pluralism. Sections 4 and 5 contain case-studies of the explanatory practice in the biomedical and social sciences, in which we develop such indispensability arguments. 


\section{Why metaphysical reductionism does not have explanatory consequences.}

In this section, we argue that explanatory reduction cannot be defended on metaphysical grounds. This is a variation on earlier discussions on the link between metaphysics and explanation (see e.g. Pettit, 1993) and not a fundamentally new insight. Nevertheless, we present our short version here for readers unfamiliar with the argument - as we need it for what follows - but without exploring all possible links between metaphysical and explanatory reduction, or discussing all possible understandings of reduction (already done by, e.g., Mayr, 1982; Sarkar, 1992).

Our argument rests on a view on the aims of science which has two elements. The first is that the aim of science is not just to provide a true description of the world. In Science, Truth and Democracy, Philip Kitcher formulates an argument supporting this view:

Nobody should be beguiled by the idea that the aim of inquiry is merely to discover truth, for, as numerous philosophers have recognized, there are vast numbers of true statements it would be utterly pointless to ascertain. The sciences are surely directed at finding significant truths. But what exactly are these? (2001: 65)

Kitcher's answer to the question at the end of the quote is double. On the one hand, there is practical significance:

One possible answer makes significance explicitly relative - the significant truths for a person are just those the knowledge of which would increase the chance she would attain her practical goals. Or you could try to avoid relativization by focusing on truths that would be pertinent to anyone's projects - the significant truths are those the knowledge of which would increase anyone's chance of attaining practical goals. (2001: 65)

But for Kitcher there is more: 
Neither of these is at all plausible as a full account of scientific significance, and the deficiency isn't just a result of the fact that both are obviously rough and preliminary. Linking significance to practical projects ignores areas of inquiry in which the results have little bearing on everyday concerns, fields like cosmology and paleontology. Moreover, even truths that do facilitate practical projects often derive significance from a different quarter. Surely the principles of thermodynamics would be worth knowing whether or not they helped us to build pumps and engines (and thereby attain further goals). Besides the notion of practical significance, captured perhaps in a preliminary way by the rough definitions given above, we need a conception of "theoretical" or "epistemic" significance that will mark out those truths the knowledge of which is intrinsically valuable. (2001: 65)

The second element of our view on the aims of science is that, while finding practically and epistemically significant truths is a legitimate aim of science, there is no reason to exclude the search for interesting fiction as an aim of science. Interesting fiction refers to theories, models, laws etc. which cannot be true (given certain other beliefs we have) nevertheless are practically or theoretically useful.

Let us first examine the explanatory consequences of weak metaphysical reductionism. An adherent of this position accepts both higher level and lower level explanations as descriptions of parts of the world. In order to arrive at explanatory reductionism, he has to argue that lower level explanations are always more significant truths than higher level explanations. In other words: it must be shown that the practical and theoretical significance of truths always increases when we go to lower levels. However, a weak metaphysical reductionist can also go the other way: he can argue that significance always decreases when we go to lower levels. Or he can argue that the significance is context-dependent and thus that there is not general tendency. In other words: a weak metaphysical reductionist can be an explanatory reductionist, a explanatory anti-reductionist or defend an intermediate position. The metaphysical position puts no restrictions on the possible explanatory positions (also see Sarkar, 1992: 169). 
Let us now look at strong metaphysical reductionism. For an adherent of this position higher level explanations are fictions while lower level explanations are truths. ${ }^{2}$ In order to arrive at explanatory reductionism, a strong metaphysical reductionist has to argue that lower level explanations always provide interesting truths, and the fictions of the higher level explanations are always uninteresting. But a strong metaphysical reductionist can also arrive at explanatory antireductionism, by arguing the lower level explanations are always uninteresting truths and that higher level explanations always are interesting fictions. And the intermediate position is also possible (argue that sometimes the higher level fictions are interesting, sometimes the lower level truths).

Similar arguments can be given for other metaphysical positions. A metaphysical emergentist position (accepting both levels as real) has no explanatory consequences because the options and the possible arguments are the same as for a weak metaphysical reductionist. A metaphysical holist (who denies the reality of lower level entities) can still be an explanatory reductionist (the options and arguments are the mirror image of the options and arguments of the strong metaphysical reductionist) (also see Van Bouwel and Weber, 2002a; Van Bouwel, 2004).

Since metaphysical positions do not restrict the possible explanatory positions one can take, we put metaphysics between brackets in the rest of the paper and provide a pragmatic argument in favour of reductive explanations: we argue for their indispensability because of their pragmatic value. While such indispensability arguments are often used to defend higher-level explanations (e.g., Jackson and Pettit, 1992), we use them to defend lower-level, reductive explanations here. The pragmatic arguments for reductionism that can be found in the literature are very underdeveloped. Take for instance Webster's argument for explanatory reductionism:

History of science shows, as a matter of fact, that reductionism works. (2003: 45)

We think there is more to be said than this. History of science only tells us that some attempts at decomposition succeeded. It does not tell us what the advantages of these decompositions are, as

\footnotetext{
${ }^{2}$ Or, alternatively, the entities of the lower level explanations are true, while the ones of the higher level explanations are fictions, because, as Hacking (1983) has defended, one may be a realist about entities without being a realist about theories. We will not develop the "realist about entities" - option here as the conclusions in relation to explanations will be similar to the ones of the "realist about theories" - option. (Thanks to an anonymous reviewer for drawing our attention to this distinction.)
} 
compared to higher-level explanations. For that, we need philosophical reflection on the history of science. This is what we do in Sections 3-5.

\section{Explanatory pluralism and epistemic interests.}

Explanatory pluralism consists in the claims that (i) the best form (and level) of explanation depends on the kind of question one is willing to answer by the explanation and (ii) that in order to answer all explanation-seeking questions in the best way possible we will need more than one form (and level) of explanation. Suppose that we observe that $x$ has property $\mathrm{P}$ at time $t$. This observation can give rise to different explanation-seeking questions - motivated by different epistemic interests - even if all questions are assumed to be requests for causal explanations, e.g.: Why does $x$ have property $\mathrm{P}$, rather than the more desirable property $\mathrm{P}^{\prime}$ ? Is the fact that $x$ has property $\mathrm{P}$ the predictable consequence of some other events? Is the fact that $x$ has property $\mathrm{P}$ causally connected with events we are more familiar with? Subsequently, the answer/explanation should be evaluated in relation to a question that is a specific request for information (and the precise formulation of the question is therefore important). Making the questions as explicit as possible will show that an apparently similar question about one phenomenon may, given that interests and contexts select distinct objects of explanation, result in very different answers providing the most adequate, efficient and accurate explanatory information (in relation to the explanatory requests and the epistemic interests motivating them). ${ }^{3}$

In earlier research we have developed arguments defending the value of higher-level explanations in history, psychology and the social sciences making the idea of explanatory pluralism philosophically explicit (cf., Van Bouwel and Weber, 2002b; Weber and Van Bouwel, 2002; Van Bouwel and Weber, 2008). We argued that the explanatory practice of scientists in these disciplines shows how different epistemic interests might lead to choosing different forms of explanation on different levels. Among these different epistemic interests for providing an explanation, we encounter therapeutic and remedial interests, prediction, straightforward curiosity, explaining unexpectedness, and so on. In what follows

\footnotetext{
${ }^{3}$ The adequacy, efficiency and accuracy of explanations are elaborated in Van Bouwel and Weber (2008).
} 
we argue that some epistemic interests require a reductive explanation, the latter providing the most adequate, efficient and accurate explanatory information (in relation to the explanatory requests and the epistemic interests motivating them), and thus indispensable following those criteria, not following any metaphysical conviction.

\section{Case studies from the biomedical sciences}

4.1 Consider the following question:

(I) Why do Belgians who spend their summer holidays in the Mediterranean area develop more often skin cancer than those who spend their holidays in Belgium?

A possible answer is that in the group of the Mediterranean holiday spenders the exposure to sun rays is higher. We call this answer an environmental explanation, because it refers to a property which the whole group has in common and that is external to the individuals in the group. In particular, it refers to the environment of the group as a whole, which stands apart from the properties of the particular individuals within that group. Consider now another question:

(II) Why do some people with high exposure to sun rays develop cancer of the skin, while others don't?

A possible answer is that some people protect themselves by using a sufficient amount of sun tan lotion, while others don't. We call this answer an individualist explanation because it refers to differences among individuals in the group - this can be differences qua behaviour, constitution, etc. Finally, consider the following questions:

(III) Why do some people who protect themselves with sun tan lotion nevertheless get cancer of the skin? 
(IV) Why do some people who do not protect themselves and spend a lot of time in the sun do not get cancer of the skin?

A possible answer is that there are genetic differences between people: some people are more susceptible to risk factors like sun rays than others. We call this answer a reductive explanation because it refers to differences among individuals in the group (so it is an individualist explanation) and on top of that refers to properties that require decomposition, contrary to the environmental and individualist (non-reductive) explanations. ${ }^{4}$

Apart from clarifying our terminology (reductive explanation - individualist explanation environmental explanation) these examples can be used to argue that a reductive account can be useful. Let us start with question (I). The attempt to answer this question leads to causal knowledge:

(A) In the Belgian population, taking summer holidays in the Mediterranean area is a positive causal factor for skin cancer.

This information is useful for prevention, because it identifies a risk factor, i.e. the higher irradiation to which the group of Mediterranean holiday spenders is exposed. Answering question (II) also results in information that is useful for prevention:

(B) Using sun tan lotion is a negative causal factor for skin cancer.

Answering questions (III) and (IV) does not result in knowledge of additional risk factors (positive causal factors) or protective measures that can be taken (negative causal factors). ${ }^{5}$ However, they lead to the identification of subpopulations in which the risk factor identified in $(A)$ is more dangerous than in other subpopulations. Analogously for the negative causal factor in (B). This information is also useful for prevention. Since questions (III) and (IV) can only be answered by means of a reductive

\footnotetext{
${ }^{4}$ Note that, in our terminology, all reductive explanations are individualist explanations, but not vice versa. Decomposition is situated within the individual here.

${ }^{5}$ Presuming that genetic differences cannot be removed (for now).
} 
analysis, our example shows that decomposition and the reductive explanations to which they lead can be useful from a practical point of view. ${ }^{6}$

4.2 The practical payoff of decomposition varies from context to context. Let us look at an example where it is very small. Suppose that public health officials ask the following question after observing an alarming amount of mesothelioma (a form of cancer that is almost always caused by exposure to asbestos) in area $\mathrm{A}$ :

(V) Why do people who live in area A develop more mesothelioma than other inhabitants of the same country?

Suppose further that the attempts to answer this question lead to the following causal knowledge:

(C) The presence of factory $\mathrm{F}$ is a risk factor for the inhabitants of area $\mathrm{A}$.

This knowledge can lead to different preventive measures: closing down the factory, reduce the level of pollution by the factory, or remove the inhabitants. In our terminology, $(\mathrm{C})$ is an environmental explanation. Now consider the following question, which the public health officials also may ask:

Why do some people in area A develop mesothelioma, while others don't?

The answer could be a reductive one, identifying genetic traits which make some people susceptible to this type of cancer, and identifying alternative genetic make-ups which protect people against mesothelioma.

In principle, this reductive analysis makes a new kind of health policy possible: genetic screening combined with an advice or obligation for some people to move out of area A. However, such a policy might be difficult to implement, e.g. because problems might arise at a later stage when

\footnotetext{
${ }^{6}$ Decomposition (and explanatory reduction) can also be linked to other (more theoretical) epistemic interests like understanding. This has been done, for instance, by Mayr (1982: 60). He considers explanatory reduction as sometimes being yielding understanding, e.g. "(...) the functioning of an organ is usually not fully understood until the molecular processes at the cellular level are clarified."
} 
people with the "right" genetic traits get children with "dangerous" genes. The macro-level policy measures seem easier to implement, so the practical relevance of the reductive analysis is limited.

4.3 As a case where the practical payoff of a reductive analysis is much higher, consider the case of Alzheimer's disease (AD). In their discussion of the causes of AD, Dekkers and Olde Rikkert (2006) present the complex causal web leading up to forms of $A D-a$ web in which neurological and genetic factors figure. Dekkers and Olde Rikkert notice moreover that the current definition and classification of $A D$ does not mention any genetic factors (2006: 278). However, several researchers focus on genetic causal factors. A first example is a successful experience of Preimplantation Genetic Diagnosis for early-onset $A D$, in which only V717L mutation free embryos were implanted in a 30-yearold woman with no signs of AD who carried the V717L mutation (Velinsky et al., 2002). A second example is the injection - with mixed success - of genetically modified skin cells that produce a protein called nerve growth factor, which prevents cell death in the brain (http://www.newscientist.com/article/dn4930.html; Tuszynski, 2007). Dekkers and Olde Rikkert (2006: 281, our italics) conclude that: "Doctors and researchers in the above examples are interested in one particular causal factor that they can influence in order to prevent AD (case 1) or to relieve the symptoms of AD (case 2)."

Let us analyse this case in a similar way as in Section 4.2. One of the questions research into the causes of $A D$ has to answer is this:

(VII) Why do some people that have been exposed to a set of risk factors R get AD, while other people subjected to the same risk factors don't get AD?

A reductive analysis, focusing on genetic traits, gives us the following answer:

(D) Early-onset AD is considered being caused by the V717L mutation, and people without this mutation cannot get early-onset $A D$ (this is the causal reasoning of Velinsky et al.).

Apart from being a maximally effective way of preventing AD, the identification by Velinsky et al. (2002) of a necessary cause has another benefit: it can be easily manipulated (contrary to what was the case in the example in Section 4.2). The combination of the two properties gives the reductive 
analysis a considerable practical relevance, addressing the epistemic interest of manipulability efficiently. (We leave the question whether this operation is legally allowed everywhere between brackets.)

4.4 Concluding this section, we want to make some general remarks. A frequent form of reduction in biomedical sciences might be labelled geneticization; it leads to viewing complex traits like cancer, schizophrenia and alcoholism, for which significant environmental and other 'non-genetic' contributions have been identified in the past, as 'genetic'. As became obvious above, this tendency of geneticization is still present. Often it has been interpreted as a consequence of scientists subscribing (in official and public contexts) to the fundamental theory within molecular genetics which sees genes as the fundamental units responsible for guiding all basic life processes. ${ }^{7}$ But, given that a lot of philosophical arguments have been formulated against that theory (cf. Waters, 2006: 190), why is the theory being upheld? One answer might be that scientists still believe in genetic determinism. Another, more plausible, answer is that so much biomedical research is centred on genes and DNA because genes are assumed to be handy handles to manipulate biological processes. The explanations in terms of genes serve some epistemic interests (cf. section 3), like manipulability, better than 'nongenetic' explanations do (see also Gannett, 1999).

Philosophers of science might have been focussing too much on science as a theory (understanding the increasing geneticization as driven by fundamental theorizing), and neglected distinct -not merely theoretical- epistemic interests. This affected the debate on reductionism as well. A more pragmatic approach towards reductionism seems more in line with practicing scientists, cf. the comments of the biologist Günter Wagner on a book of a philosopher of biology, i.e., Jason Scott Robert: "Robert and his colleagues who argue for a similar interpretation are correct in that the genes alone cannot make an organism and instead are embedded in a large network of causal interactions. But scientists are usually not interested in general statements about what in principle is required to understand a phenomenon. (...) Yes, scientists make pragmatic decisions about what to study, but I think that these

\footnotetext{
${ }^{7}$ The fundamental theory should be distinguished from the basic theory. Where the basic theory is more modest in answering to the question 'What do genes do?', i.e., they "code for" or "determine" the linear sequences in RNA molecules and polypeptides synthesized in the cell, the fundamental theory is bolder, claiming that genes are "fundamental" entities that "direct" the development and functioning of organisms by "producing" proteins that in turn regulate all the important cellular processes. (Waters, 2007)
} 
decisions are anything but arbitrary. The power of molecular genetic approaches did not come easily; rather it is the result of a long history of strenuous research based on a vision that derived from the work of Richard Goldschmidt, Alfred Kühn, and Thomas Hunt Morgan early in the 20th Century. It is thus not intellectual laziness that drives the genetic research program; instead we are raking in the spoils of a hard-won victory over biological complexity." (Wagner, 2004: 1405)

Thus, the pragmatic aspects of reduction should be taken into account by philosophers that aim to elucidate scientific explanation. As this section has shown, for a researcher in medical science (as well as for a patient and the ones taking care of her) the interest might often be in identifying the necessary cause - gene(s) - and eliminate it (preventive or curative) rather than focus on the sufficient conditions in which the genetic defect manifests itself. The gene as such is hardly ever sufficient, but from a pragmatic point of view it might often be preferable (more effective) to change the gene than the variety of environmental factors making the genetic defect manifest itself. Hence, reductive explanations are indispensable in providing the most adequate, efficient and accurate explanatory information (in relation to the explanatory requests and the epistemic interests motivating them).

\section{Examples from the social sciences.}

5.1 In the social sciences, one can identify similar strategies of reduction. In designing social policies to deal with problems like crime and violence, a scientist might, for instance, follow the strategy of biologisation by focussing on causes of crime, violence and aggression in the biological sphere (the causal space of biology, e.g. genetic, hormonal factors), or instead focus on the social sphere (environmental factors, like family, neighbourhood, unemployment, ...). ${ }^{8}$ Metaphysical reductionists would find explanations in biological terms always superior to explanations in social terms (and reject environmental explanations of criminal behaviour). In what follows, we will illustrate how our approach to explanations can identify cases in which formulating a reductive explanation is the best strategy, and other cases in which it is not. Let us start with the following general explanation-seeking question:

\footnotetext{
${ }^{8}$ Famous examples of biologisation in social science are E.O. Wilson's (1975) Sociobiology: The New Synthesis, and sociobiology's heir, evolutionary psychology.
} 
(VIII) Why do we have high crime rates in American society?

According to the institutional anomie theory (which furthers ideas of Robert Merton, 1938 and 1968) criminal behaviour can be explained by an environmental explanation referring to social factors:

(E) Criminal behaviour is caused by the contradictions and disjunctions within the culture, and between the culture and a central feature of the social structure, the stratification system.

This is the case within American culture, according to Merton. He detects a disproportionate emphasis on the importance of realizing cultural goals, especially the goal of monetary success ("the American Dream") relative to the emphasis placed on the importance of using the legitimate means for pursuing goals. This cultural imbalance has created a "cheating orientation" and fosters anomie, wherein the means for social action are selected purely on the basis of considerations of technical efficiency. Furthermore, according to Merton, a structural strain is built into the social organization of American society derived from the combination of universal success goals and unequal access to the legitimate means for realizing success. (Messner and Rosenfeld, 2006: 128)

Merton's approach, explaining macro-level phenomena such as crime rates by referring to the fundamental features of the social organization of a society, was supported by Messner and Rosenfeld's (1994), testing the utility of institutional anomie theory (IAT) for predicting crime rates across aggregate units and finding that crime rates can be predicted depending on structural factors within society (see also, Maume \& Lee, 2003: 1137; and for more empirical literature supporting IAT, Messner and Rosenfeld, 2006: 137).

5.2 But the general explanation-seeking question (VIII) can be made more specific:

(IX) Why does criminal or deviant behaviour manifests itself in American-born students A, B, C, but not in foreign-born $\mathrm{X}, \mathrm{Y}, \mathrm{Z}$ ?

Lisa Muftic (2006) tested the robustness of institutional anomie theory by operationalizing IAT variables at the individual level and looking at a minor form of deviance, student cheating. This 
approach envisages explaining differences among individuals, i.e., undergraduate students, Americanborn and foreign-born, surveyed about their cheating behaviours and adherence to economic goal orientations. Muftic (2006: 648$)$ finds that:

(F) Students with higher adherence to the cultural values of universalism and the fetishism of money have a higher likelihood of cheating. Moreover, location of birth (i.e., born in the United States) appears to have the strongest impact on cheating, regardless of the statistical model used.

Here we have an individualist explanation (cf. our distinctions in section 4.1) answering the question (IX). Besides the American cultural values as explanation for criminal or deviant behaviour, differences in gender and age do seem to play a (statistical) role (see Table 6, p. 646 in Muftic 2006). Muftic does not focus on these differences, but others have done so.

5.3. Lee Ellis (2005) develops an evolutionary neuroandrogenic theory (ENA) to account for statistical associations between biological variables and criminal behaviour. His theory is an instance of biologisation and can address questions like:

(X) Why does person A manifest criminal behaviour, while B does not (even though A and B share the same social environment)?

ENA theory explains the differences qua criminal behaviour by referring to biological differences; it can explain, for instance, the concentration of offending among males between the ages of 13 and 30, a pattern that, according to Ellis, strictly environmental theories, like IAT, have always had difficulty explaining.

Ellis's ENA theory rests on two under-girding propositions. The first, an extension of Darwin's theory of evolution, states "that males on average exhibit competitive/victimizing behaviour more than females because females who prefer to mate with such males increase their chances of having mates who are competent providers of resources. These female choices have evolved because females who have 
had the assistance of competent providers have left more offspring in subsequent generations than other females. No comparable reproductive advantage comes to males who select mates based on resource procurement capabilities." (Ellis, 2005: 305) This competitive/victimizing behaviour comes in different forms, some are crude - requiring little skill or learning - and nearly all of these are assaultive or confiscatory behaviours; this form of behaviour is condemned by most people in all societies. Other forms are more sophisticated involving much more subtle types of victimization, which arise, for instance, "in the context of profitable business ventures and the management of large organizations" (Ibid.) and are, in most societies, tolerated and indeed encouraged.

The second proposition under-girding ENA theory is that "genes that have evolved on the Ychromosome cause male brains to develop differently from female brains, leading to higher rates of competitive/victimizing behaviour. These genes operate in part by causing would-be ovaries to develop instead into testes early in foetal development. Once differentiated, the testes produce testosterone and other sex hormones, which have three hypothesized effects upon brain functioning, all of which promote competitive/victimizing behaviour. The three effects are termed suboptimal arousal, proneness to seizures, and a rightward shift in neocortical functioning. Furthermore, two neurological processes are hypothesized to help individuals shift from crude to sophisticated forms of competitive/victimizing behaviour. These are learning ability (or intelligence) and executive cognitive functioning (or planning ability). The higher or more efficient these latter two traits are, the more quickly individuals will shift from crude to sophisticated forms of the behaviour." (Ellis, 2005: 305-306; furthermore, Ellis reviews evidence on links between criminality and biological variables, like testosterone, mesomorphy, cortisol, serotonin, monoamine oxidase, hypoglycemia, maternal smoking during pregnancy, epilepsy, altered heart rate and skin conductivity, and certain brainwave patterns.)

Given these elements, the explanation-seeking question $(\mathrm{X})$ could be answered as follows:

(G) The biological characteristics of person A (e.g., male sex hormones operating on the human brain, aged between 13 and 30), not shared by person B, increase the probability of competitive/victimizing behaviour and, hence, criminal behaviour. 
As already mentioned competitive/victimizing behaviour is "hypothesized to exist along a continuum, with 'crude' (criminal) forms at one end and 'sophisticated' (commercial) forms at the other. Individuals with the greatest capacities to learn and plan will move rapidly after puberty from criminal to noncriminal forms of competitive/victimizing behaviour." (Ellis, 2005: 287) We notice in this last phrase that Ellis theory leaves some space for environmental factors. Persons with a certain biological set-up are not necessarily doomed to become and remain criminals; the right stimuli to improve planning and learning can make a rapid move from criminal to non-criminal forms of competitive/victimizing behaviour more likely. (However, Ellis dedicates very few lines to the non-biological factors, as one could expect from a pragmatic reductionist.)

5.4 Ellis' reductive explanation does provide us with an answer to questions like (X), an answer we cannot obtain from IAT, as the latter does not explain age- or gender-differences. Questions like (VIII), on the contrary, can be answered by IAT in a more efficient and adequate manner than ENA. Thus, following a pragmatic theory of explanation, the debates concerning explanations in social science should not focus (as they very often do, cf. the debates between sociobiologists and their critics) on whether it are the reductive biological accounts or anti-reductive social accounts which always provide the better explanations. They should rather focus on the explanatory information required, on the explanation-seeking question to be answered. Our pragmatic approach to explanations provides a clear alternative to the often fruitless discussions of social scientists and philosophers about what the best way of explaining would be - presupposing that there would be one single model or type of explanation or one theoretical perspective that always prevails and has to be applied for all questions concerning social phenomena.

We have illustrated that in dealing with the plurality of explanations of criminal behaviour, we need first of all philosophical instruments to make the explananda as explicit as possible, paying attention to the underlying epistemic interests (cf. section 3). This is imperative in order to avoid considering two explanations as being competitors, while they actually have different explananda. Secondly, starting from specific explanation-seeking questions, it was shown that a winner-takes-all-approach to explanations in social science (where it seems that the successfulness of a form of explanation in one particular field, or in relation to one particular question, suffices to claim that it should be used in all 
possible fields in the social sciences) is not tenable, and should be replaced by a pragmatic approach towards social explanation which allows for different forms of explanations and theories, elaborating a framework for explanatory pluralism.

Defences of explanatory pluralism generally focus on proving the indispensability of higher-level explanations (cf. Van Bouwel, 2004). We have extended this to reductive explanations, not defending them (as usually is done) on metaphysical grounds, but on pragmatic grounds. Which level of explanation is the better one depends on the explanation-seeking question that has to be answered questions and answers with significant differences in structure and motivation. The differences qua explanatory information provided by the different forms of explanation (i.e., environmental , individualist and reductionist) suggest that we should not keep on seeking for one comprehensive model or form for explanations across all (social) sciences. Focusing on social scientific practice, it is fruitful to make the explanandum more explicit and to start analyzing the advantages of different models relative to different epistemic interests, to the different problems to be solved-a pragmatic approach. The study of scientific explanation would then be more local and contextual, concentrating on the explanation-seeking question at hand, and analyzing which epistemic interests are decisive. ${ }^{9}$

What could that imply for the relations or interactions between different theoretical perspectives in the social sciences? Returning to our example of explaining criminal behaviour, different theoretical approaches tackling criminal and aggressive behaviour can be distinguished, for instance, behaviour genetics, social-environmental approaches (behavioural dispositions of socialization patterns, familial environments, and/or parental attitudes and interactions with their children), neurobiology (physiology and anatomy - the neural substrate of behaviour) and developmental systems. Where these different theories are often understood as rival claims to nothing but the truth, intending to substitute one another, one would better focus on the different kinds of explanatory information they can provide us with - spelling out the limitations and partiality of these approaches by emphasising that they do tackle different questions (although there seems to be the presupposition that they are all asking the same questions) and by pointing at the different ways in which the causal space is parsed and causal relations conceptualized. In her Theoretical Pluralism and the Scientific Study of Behavior (2006),

\footnotetext{
${ }^{9}$ Worries of relativism might arise here. We refer the reader to Van Bouwel and Weber (2008), which explicitly discusses the question of how to avoid relativism in a pluralistic framework.
} 
Helen Longino does exactly that and concludes that: "some of the assumptions differentiating these approaches from each other involve differences so deep as to make their unification, whether through integration or through the elimination of all but one compatible set of hypotheses and approaches, impossible." (Longino, 2006: 117) This differs from a widely held belief that theories, models or representations in general that conform to nature are jointly consistent - a monist metaphysical prejudice, that brings us to discussing metaphysical fallacies.

\section{Conclusion.}

The epistemological and methodological problems concerning the form and level of explanation are often considered to be essentially metaphysical problems. Instances of explanatory reduction are thus defended on metaphysical grounds; given that the only real things in the world are subatomic particles and their interaction, we have to try to explain everything in terms of the laws of physics. In this paper, we have shown that explanatory reduction cannot be defended on metaphysical grounds. Nevertheless, indispensability arguments for reductive explanations can be developed taking into account actual scientific practice and the role of epistemic interests. Reductive explanations might be indispensable to address some epistemic interest answering a specific explanation-seeking question in the most accurate, adequate and efficient way. Just like explanatory pluralists often advocate the indispensability of higher levels of explanation pointing at the pragmatic value of the explanatory information obtained on these higher levels, explanatory reduction - traditionally understood as the contender of pluralism - can be defended in a similar way.

In the above examples, defenders of reductive explanations can find support for their position, but so can pragmatic explanatory pluralists. The importance of genetic or molecular details to address some specific epistemic interests in the biomedical sciences, attracts the attention of reductionists; pluralists will emphasise the non-genetic and non-molecular remedies (which put the heterogeneity of underlying molecular levels between brackets) to be found on higher levels (cf. De Vreese et al., 2010). In the social sciences, reductive explanations invoking biological factors can address questions (and epistemic interests) left unanswered by higher-level explanations. Hence, the instances of 
reduction presented in this paper do not lead us to defending reduction as a regulative ideal of science (i.e., science should aim at lower-level explanations, reducing as far as possible), but rather to emphasising the importance of making epistemic interests and the different questions they induce, as explicit as possible. Taking into account distinct epistemic interests elucidates the possible advantages of reduction in the practice of the biomedical and social sciences. Distinct interests lead to distinct explanation-seeking questions, and, answering these questions, we notice that sometimes reduction does provide us with the most adequate answer, sometimes not; that is why they are indispensable.

\section{References.}

Dekkers, W. and M. Olde Rikkert (2006) 'What is a genetic cause? The example of Alzheimer's Disease.' Medicine, Health Care and Philosophy 9: 273-284.

De Vreese, L., E. Weber and J. Van Bouwel (2010). 'Explanatory pluralism in the medical sciences: Theory and practice.' Theoretical Medicine and Bioethics 31(5): 371-90.

Ellis, L. (2005) 'A Theory Explaining Biological Correlates of Criminality.' European Journal of Criminology 2(3): 287-315.

Gannett, L. (1999) 'What's in a cause?: The pragmatic dimensions of genetic explanations.' Biology and Philosophy 14(3): 349-374.

Hacking, I. (1983) Representing and Intervening. Cambridge: Cambridge University Press. Jackson, F. and P. Pettit (1992) 'In defense of explanatory ecumenism.' Economics and Philosophy 8: $1-21$.

Kitcher, Ph (2001) Science, Truth and Democracy. New York: Oxford University Press Longino, H. (2006) 'Theoretical Pluralism and the Scientific Study of Behavior.' In: Kellert, S,, H. Longino, and K. Waters (eds.) Scientific Pluralism. Minnesota Studies in the Philosophy of Science XIX. Minneapolis: University of Minnesota Press, pp. 102-131.

Maume, M.O. and M.R. Lee (2003) 'Social institutions and violence: A sub-national test of institutional anomie theory.' Criminology 41(4): 1137-1171.

Mayr, E. (1982) The growth of biological thought. Diversity, evolution, and inheritance. Cambridge, MA: Belknap Press. 
Merton, R.K. (1938) Social structure and anomie. American Sociological Review, 3, 672-682.

Merton, R.K. (1968) Social theory and social structure. Glencoe, IL: Free Press.

Messner, S.F. and R. Rosenfeld (1994) Crime and the American Dream. Belmont, CA: Wadsworth

Messner, S.F. and R. Rosenfeld (2006) 'The Present and Future of Institutional-Anomie Theory.' In:

Cullen, F.T., J.P. Wright, K.R. Blevins (eds.) Taking Stock: The Status of Criminological Theory. New Brunswick, NJ: Transaction Publishers, pp. 127-148.

Muftic, L.R. (2006) 'Advancing Institutional Anomie Theory: A Microlevel Examination Connecting Culture, Institutions, and Deviance.' International Journal of Offender Therapy and Comparative Criminology 50(6): 630-653.

Pettit, P. (1993). The Common Mind. Oxford: Oxford University Press.

Sarkar, S. (1992) 'Models of reduction and categories of reductionism' Synthese 91: 167-194.

Tuszynski, M. (2007) 'Nerve growth factor gene delivery: Animal models to clinical trials.' Developmental Neurobiology 67(9): 1204-1215.

Van Bouwel, J. (2004) 'Individualism and Holism, Reduction and Pluralism.' Philosophy of the Social Sciences 34(4):527-535.

Van Bouwel, J. and E. Weber (2002a) 'The living apart together relationship of causation and explanation.' Philosophy of the Social Sciences 32(4): 560-569.

Van Bouwel, J. and E. Weber (2002b) 'Remote causes, bad explanations?' Journal for the Theory of Social Behaviour 32(4): 437-449.

Van Bouwel, J. and E. Weber (2008) 'A pragmatic defence of non-relativistic explanatory pluralism in history and social science.' History and Theory 47: 168-182.

Velinsky, Y. et al. (2002) 'Preimplantation diagnosis for early-onset Alzheimer disease caused by V717L mutation.' Journal of the American Medical Association 287:1018-1021.

Wagner, G. (2004) 'Review of Embryology, Epigenesis, and Evolution: Taking Development Seriously, by Jason Scott Robert.' Science 305: 1405-1406

Waters, C. K. (2006) 'A Pluralist Interpretation of Gene-centered Biology.' In S. Kellert, H. Longino, and C. K. Waters (eds.) Scientific Pluralism, Volume XIX, Minnesota Studies in the Philosophy of Science, Minneapolis: University of Minnesota Press.

Waters, C. K. (2007) 'Molecular Genetics.' In: The Stanford Encyclopedia of Philosophy. 
Weber, E. and J. Van Bouwel (2002) 'Can we dispense with the structural explanation of social facts?' Economics and Philosophy 18: 259-275.

Webster, S. (2003) Thinking about Biology. Cambridge: Cambridge University Press.

Wilson, E.O. (1975) Sociobiology: The New Synthesis. Cambridge, MA: Belknap Press. 